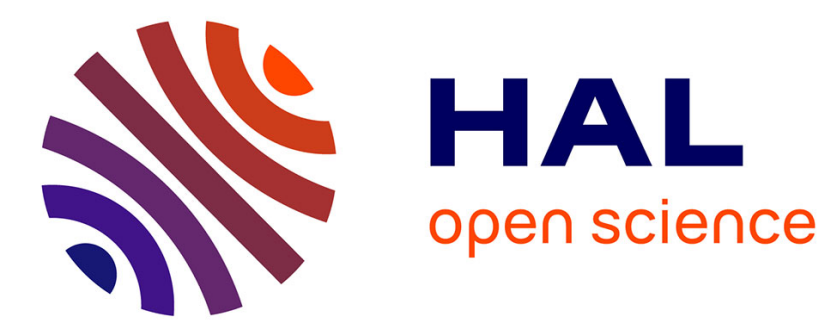

\title{
Free electron laser infrared irradiation of molecules isolated in matrices as a tool for molecular relaxation studies
}

\author{
P. Roubin, J. Berset, S. Cardonatto, H. Dubost, J. Lefèvre, J. Ortéga
}

\section{- To cite this version:}

P. Roubin, J. Berset, S. Cardonatto, H. Dubost, J. Lefèvre, et al.. Free electron laser infrared irradiation of molecules isolated in matrices as a tool for molecular relaxation studies. Journal de Physique IV Proceedings, 1994, 04 (C9), pp.C9-385-C9-392. 10.1051/jp4:1994964 • jpa-00253527

\section{HAL Id: jpa-00253527 https://hal.science/jpa-00253527}

Submitted on 1 Jan 1994

HAL is a multi-disciplinary open access archive for the deposit and dissemination of scientific research documents, whether they are published or not. The documents may come from teaching and research institutions in France or abroad, or from public or private research centers.
L'archive ouverte pluridisciplinaire HAL, est destinée au dépôt et à la diffusion de documents scientifiques de niveau recherche, publiés ou non, émanant des établissements d'enseignement et de recherche français ou étrangers, des laboratoires publics ou privés. 


\title{
Free electron laser infrared irradiation of molecules isolated in matrices as a tool for molecular relaxation studies
}

\author{
P. Roubin, J.M. Berset*, S. Cardonatto, H. Dubost**, J. Lefèvre** and J.M. Ortéga* \\ P2IM, URA 773, Université de Provence, 13397 Marseille cedex 20, France \\ * LURE, Bât. 209D, Université Paris-Sud, 91405 Orsay cedex, France \\ ** LPPM, Bât. 213, Université Paris-Sud, 91405 Orsay cedex, France
}

\begin{abstract}
The selective excitation of different vibrational modes of the $\mathrm{CH}_{2} \mathrm{D}-\mathrm{CH}_{2} \mathrm{D}$ molecule isolated in a xenon matrix at $7 \mathrm{~K}$ has been performed using the free electron laser source CLIO. The aim of these experiments was to test the selectivity of the different intramolecular couplings involved in the isomerization induced by the infrared irradiation. Kinetics measurements have been recorded using FT-IR spectroscopy as a probe of the sample composition and the modifications of the quantum yield with the excited mode have been obtained. Preliminary results show that the local environment strongly influences the reaction rate. Although the overall behavior of the quantum yield with energy is consistent with a RRKM process, a non statistical redistribution of the energy among the different modes is observed in some cases.
\end{abstract}

\section{INTRODUCTION}

The knowledge of intramolecular energy transfer is one of the key in the understanding of molecular behavior and chemical reactivity. We focus our attention to unimolecular reactions which are well adapted for studying the intramolecular potential. The crucial point is to determine if the randomization of the internal energy (RRKM behavior) is in competition with mode-specific effects. A non statistical behavior would indeed allow the development of a selective photochemistry.

The isolation of molecules in cryogenic matrices is a powerful tool for spectroscopic, dynamic or relaxation studies. A mixture of the molecule of interest with a solvent is deposited on a cold window (4 $50 \mathrm{~K}$ ) and forms a crystal. The ratio can be typically varied from $1 / 100$ to $1 / 10000$. The solvent (usually a rare gas or a diatomic molecular gas) is assumed to weakly interact with the molecule and in a first approximation the spectroscopy is that of a cold and isolated molecule. At these low temperatures, kinetics are almost frozen : the trapping of unstable species is possible and this offers the opportunity of studying reaction intermediates, unstable isomers... Moreover, this technique allows the direct measurement of vibration-vibration (V-V) energy transfer time constants by pump-probe experiments. We will describe one way of obtaining information on the relaxation processes of polyatomic molecules. 
In this paper, we study the infrared induced isomerization of a deuterated derivative of ethane $\mathrm{CH}_{2} \mathrm{D}$ $\mathrm{CH}_{2} \mathrm{D}$. The selectivity of the photoinduced process can be tested by changing the excited molecular mode and this has been achieved owing to the CLIO laser at LURE (Orsay, France). In part 2, we will briefly describe the background of this study. Experimental details are developed in part 3. Results are presented in part 4 and a discussion is following in part 5. Part 6 summarizes some concluding remarks.

\section{BACKGROUND}

\subsection{Molecular Vibrations and Crystalline Environment}

In the harmonic approximation, the atomic displacements of a non linear molecule can be described by $3 N-6$ vibrational modes. They consist in in-phase motion of all the atoms with specific amplitude for each mode. The different modes are independent. Because of the existence of higher order terms (anharmonic terms) in the potential energy, energy can flow from one mode to another by specific molecular coupling. If the couplings are strong and if the density of vibrational states is important, one can assume that the energy is randomized among all the modes (RRKM hypothesis).

An important tool for studying the vibrational characteristics of molecules is of course the infrared spectroscopy. The crystalline environment induces modifications on the infrared spectra of molecules. First, the vibrational spectrum observed in the mid-infrared region is a pure one, i.e. a rotationless spectrum. The spectral interpretation is therefore easier. This is true for large polyatomic molecules $>5$ atoms) whereas small molecules exhibit an hindered rotation spectrum. Small shifts of the vibrational frequencies are also measured (a few $\mathrm{cm}^{-1}$ ) and these shifts depend on the nature of the crystal. At last, inhomogeneous broadening is frequently observed due to the existence of multiple trapping sites. The widths of the lines are ranging from 0.2 to $10 \mathrm{~cm}^{-1}$. These trapping sites can be one hole- or two holesubstitutional sites. They can be related to a fcc or a hep structure of the crystal. They can also be due to different orientations of the trapped molecule in a same environment.

Modifications concerning the relaxation phenomena are also expected. The radiative lifetime of a level is modified by the dielectric properties of the solid and is about .3 to .6 times that of the gas phase [1]. It ranges typically from 10 to $100 \mathrm{~ms}$. The homogeneous lifetime is determined by the relaxation with phonons. Usually, the molecular levels are high energy levels $\left(600-3000 \mathrm{~cm}^{-1}\right)$ while phonon energies range from 10 to $90 \mathrm{~cm}^{-1}$. The phonons thus make a thermal bath that allows the relaxation of the molecular level. This relaxation is commonly modeled in two steps : the first one is a rapid intramolecular V-V transfer that allows a downward cascading of the vibrational ladder, the second step being a multi-phonons or multi-J (if any rotation occurs) relaxation for the lower vibrational level (typically $600 \mathrm{~cm}^{-1}$ ). The first step is faster and faster as the number of atoms in the molecule grows. Legay [1] has pointed out the importance of the low-frequency localized modes (as the hindered rotation) in the relaxation processes. They are indeed more coupled with the molecular motion than the phonons. 


\subsection{Reactivity in Matrices}

The success of the technique of isolation in inert solid matrices began in 1960 with the pioneer work of George C. Pimentel [2]. The infrared photoisomerization of the nitrous acid (passage from the cis stable form to the trans unstable form) was reported, with a light in the range $3200-3600 \mathrm{~cm}^{-1}$. The vO-H vibration was excited, then, via intramolecular couplings, the rotation around the $\mathrm{N}-\mathrm{O}$ bond was excited too and this leads to the interconversion. A lot of similar studies have followed and, about 20 years later, P. A. McDonald and J. S. Shirk [3] have showed that the photoinduced cis $\rightarrow$ trans process was about 200 times more efficient than the reverse one, the same kind of vibration being excited. It was interpreted as due to the presence of an hydrogen bond like interaction between $\mathrm{O}$ and $\mathrm{H}$ in the cis form that enhances mixing of the $\mathrm{vO}-\mathrm{H}$ vibration with the rotational motion and greatly facilitates the energy transfer. In this case, the detail of the intramolecular potential is necessary to understand the phenomena and it cannot be interpreted with simple energetic or statistical arguments.

Numerous studies have been performed to answer the question : is it possible to prepare a molecule by a selective irradiation to induce a specific reaction? Many examples of unimolecular or bimolecular reactions for species embedded in cryogenic matrices have been investigated [for review, see 4-6]. In most cases a clear evidence for either a statistical process or a mode-specific process is difficult to obtain. One reason is the lack of a tunable monochromatic source in the mid-infrared region that would permit to explore the different vibrational excitations. The CLIO facility gives a new impetus to these studies.

\section{EXPERIMENTAL}

\subsection{The CLIO Laser}

The first laser oscillation on the CLIO infrared free electron laser (FEL) was obtained in January 1992 at $8 \mu \mathrm{m}$ [7]. This FEL uses a $32 / 50 \mathrm{MeV}$ rf linear accelerator and a 48 period planar undulator. The FEL optical cavity is $4.8 \mathrm{~m}$ long and uses broad band silver coated mirrors. The light is partially extracted from the cavity by a $\mathrm{ZnSe}(\lambda=0.6-18 \mu \mathrm{m}$, extraction coefficient : $1.5 \%)$ or $\mathrm{CaF}_{2}$ plate $(\lambda=0.1-10$ $\mu \mathrm{m}$, extraction coefficient $: 0.2 \%$ ), near the Brewster incidence. The infrared beam is linearly polarized and has a temporal structure consisting of micropulses (length : $0.5-6 \mathrm{ps}$, repetition rate : 8,16 or $32 \mathrm{~ns}$ ) and macropulses (length : about $8 \mu \mathrm{s}$, repetition rate : $6.25-50 \mathrm{~Hz}$ ). It allows kinetic measurements with characteristic times ranging from sub picosecond to nanosecond. The spectral range is $1.8-17.5 \mu \mathrm{m}$ with a typical spectral width of .4\%. Peak power ranges from 1 to $8 \mathrm{MW}$ while the mean power ranges from $10 \mathrm{~mW}$ to a few W, depending on the two repetition rates of the pulses. Figure 1 shows the laser power as a function of the laser wavelength for 32,40 and $50 \mathrm{MeV}$ [7].

The cavity length as well as the mirror tilts are adjusted by stepping motors, monitored from the diagnostic room by computer. This computer also allows to control the undulator gap. The wavelength can therefore easily be modified by the users. 


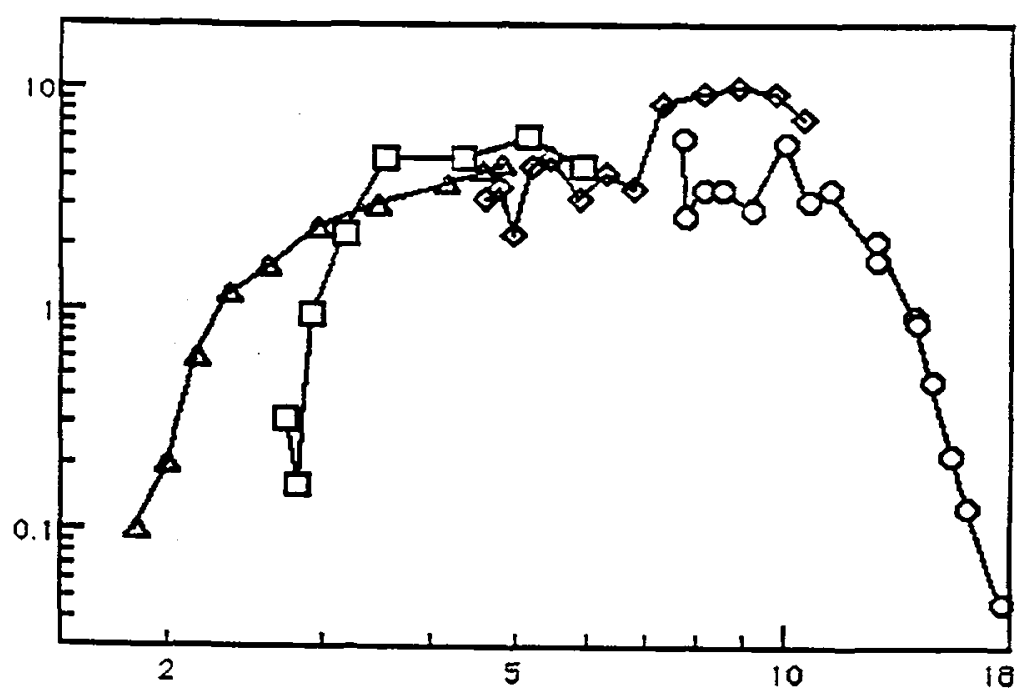

Figure 1 : Peak laser power (MW) as a function of the laser wavelength $(\mu \mathrm{m})$ for $32 \mathrm{MeV}(0), 40 \mathrm{MeV}$ $(\diamond), 50 \mathrm{MeV}(\square)$ and $58 \mathrm{MeV}(\Delta)$ electron energy.

\subsection{The Experimental Set-up}

The sample was prepared by the deposition on a gold coated block cooled at $30 \mathrm{~K}$ in a liquid helium cryostat of a mixing of $\mathrm{CH}_{2} \mathrm{D}-\mathrm{CH}_{2} \mathrm{D}$ and $\mathrm{Xe}$ at the ratio : 1/500. The matrix was optically thin and about $4 \mathrm{~cm}^{2}$ large and $10 \mu \mathrm{m}$ thick. The sample was analyzed with an FT-IR spectrometer (Mattson, serie Galaxy 7000) at a resolution of $0.5 \mathrm{~cm}^{-1}$. The infrared beam was coming on the sample through a $\mathrm{CaF}_{2}$ window at normal incidence and the recorded spectrum ranges from 1100 to $3300 \mathrm{~cm}^{-1}$. The laser beam was impinging the sample at $45^{\circ}$ of incidence through a $\mathrm{ZnSe}$ window and was leaving the cryostat through a symmetric window.

\subsection{The Molecule $\mathrm{CH}_{2} \mathrm{D}-\mathrm{CH}_{2} \mathrm{D}$}

The molecule 1,2- dideutero ethane has two forms : gauche (symmetry $\mathrm{C}_{2}$, two enantiomeric molecules) and trans (symmetry $\mathrm{C}_{2 \mathrm{~h}}$ ). One form is deduced from the other by a relative rotation of the $\mathrm{CH}_{2} \mathrm{D}$ groups of $120^{\circ}$ around the $\mathrm{C}-\mathrm{C}$ bond (internal rotation). They are both stable at room temperature and are present in the matrix at the ratio $2: 1$. Their physical properties are expected to be very close to those of the pure ethane molecule. In particular, the internal rotation potential presents a barrier height of about $1000 \mathrm{~cm}^{-1}$ [8-9]. The vibrational motion related to this barrier is the $v_{4}$ mode of torsion around the $\mathrm{C}-\mathrm{C}$ bond at 254 $\mathrm{cm}^{-1}$. The different mass distribution of the two conformers allows their clear spectroscopic identification. 


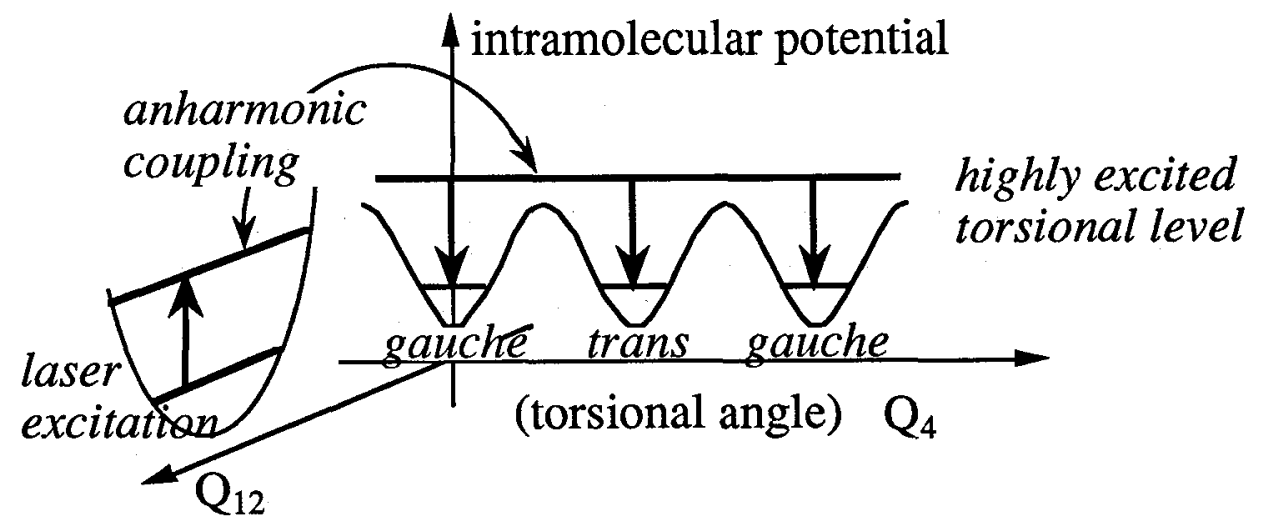

Figure 2 : Schema of the coupling between the $v_{12}$ and the $v_{4}$ modes of $\mathrm{CH}_{2} \mathrm{D}-\mathrm{CH}_{2} \mathrm{D}$.

( $\mathrm{Q}_{12}$ and $\mathrm{Q}_{4}$ are the normal coordinates related to these modes.)

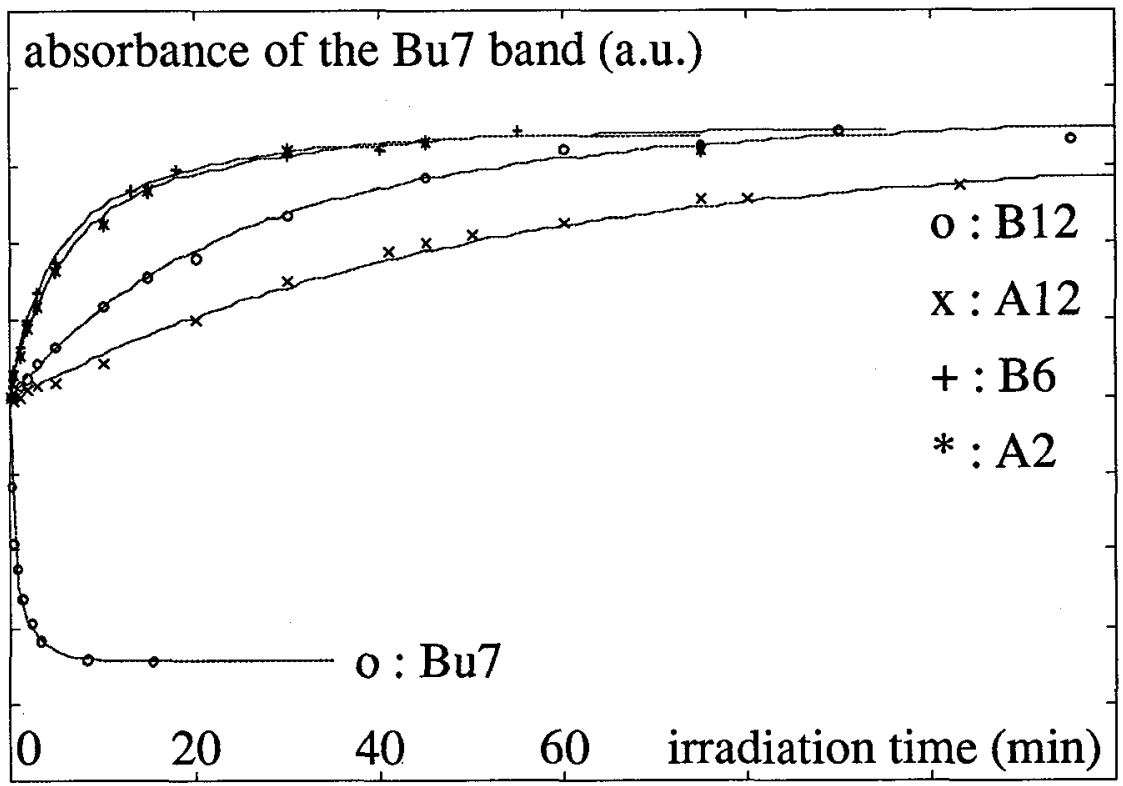

Figure 3 : Integrated absorbance of the $\mathrm{Bu} 7$ band of the trans molecule as a function of the irradiation time for different excitations (the value of the absorbance at initial time has been subtracted). 


\section{PHOTOISOMERIZATION}

\section{1. $\mathrm{CO}_{2}$ Laser Irradiation}

The resonant excitation of the $\mathrm{B} 12$ mode of the gauche form at $1047 \mathrm{~cm}^{-1}$ with the $\mathrm{P} 20$ line of a $\mathrm{CO}_{2}$ laser leads to the decay of the gauche form and to the corresponding increase of the trans form [10]. This reveals a strong intramolecular coupling between the $v_{12}$ first excited level and a highly excited torsional level of the gauche form as represented on the schema of figure 2. The aim of the experiments performed with the CLIO laser was to investigate the selectivity of this coupling by testing the excitation of different vibrational modes of both forms.

\subsection{CLIO Laser Irradiations}

The resonant excitations of the B12 $\left(1047 \mathrm{~cm}^{-1}\right), \mathrm{A} 12\left(1113 \mathrm{~cm}^{-1}\right), \mathrm{B} 6\left(1305 \mathrm{~cm}^{-1}\right), \mathrm{A} 2\left(1318 \mathrm{~cm}^{-1}\right)$ modes of the gauche form and of the Au8 $\left(1278 \mathrm{~cm}^{-1}\right)$ and Bu7 $\left(2184 \mathrm{~cm}^{-1}\right)$ modes of the trans form have been successfully performed. Except for the Au8 mode, they have led to the net decrease of the irradiated form and to the related increase of the other form. The difference spectra are well correlated in the whole infrared domain and no other feature appears. The back reaction was performed by heating the sample a few minutes at $30 \mathrm{~K}$. All the results presented here have been recorded with the same sample.

Kinetic measurements have been performed in order to determine the reaction rate and the quantum yield of the isomerization $(\phi)$. Figure 3 represents the evolution of the integrated absorbance of the $\mathrm{Bu} 7$ band as a function of the irradiation time for the different excitations (B12, A12, B6, A2 and Bu7). On the other hand, the Au8 excitation has led to a surprisingly weak reaction rate.

\section{DISCUSSION}

\subsection{Modeling}

We propose to describe the kinetics of the system using the simple mechanism shown in figure 4 in the case of the excitation of the gauche form. We consider a one photon excitation $G \rightarrow G^{*}$ (at the rate $p$ ), with elementary first order steps leading to the relaxation to the gauche or the trans form via a highly excited torsional level $\mathrm{X}^{*}$. If we apply the hypothesis of a stationary-state for the excited species, it is easy to show that the kinetics are equivalent to a simple exponential decay for the excited species. The reaction rate $\mathrm{k}$ is then given by $: \mathrm{k}=\mathrm{k}_{0}+\frac{\mathrm{n}}{3} \cdot \phi \cdot \mathrm{p}: \mathrm{k}_{0}$ is the rate of gauche $\leftrightarrows$ trans spontaneous reaction due to the ambient radiation, $\mathrm{n}=1(\mathrm{n}=2)$ for the gauche (trans) excitation and $\phi=\frac{\mathrm{kE}_{\mathrm{E}}}{\mathrm{k}_{\mathrm{R}}+\mathrm{k}_{\mathrm{E}}}$.

\subsection{Results}

The fit of the data points by a single rate kinetic law leads to a poor result compared to the fit with a twoexponential law (continuous lines of figure 3 ). These rates have then been normalized in order to take into account the influence of $\mathrm{p}$ which is proportional to the spectral density of photons coming through the sample and to the absorption cross section of the excited mode. Table 1 summarizes the values of the corresponding quantum yields $\phi_{1}$ and $\phi_{2}$. 


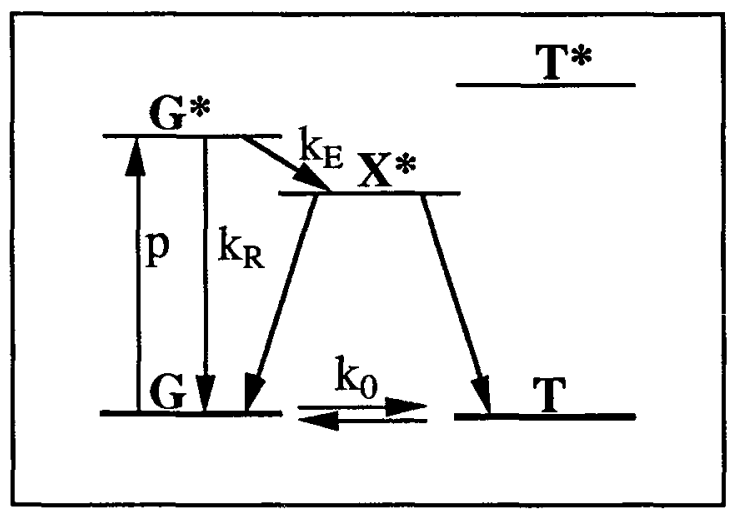

Figure 4 : Elementary steps for the kinetic modeling in the case of the laser excitation of the gauche form. $\mathrm{G}$ and $\mathrm{G}^{*}$ ( $\mathrm{T}$ and $\mathrm{T}^{*}$ ) label the fundamental and the excited level of the gauche (trans) form.

Table 1 : Normalized quantum yields $\phi_{1}$ and $\phi_{2}$ ( $\phi_{1}=1$ for the Bu7 excitation).

The experimental accuracy is $\Delta \phi / \phi \approx 0.3$.

\begin{tabular}{|c|c|c|c|c|c|}
\hline Excited mode & $\mathrm{B} 12$ & $\mathrm{~A} 12$ & $\mathrm{~B} 6$ & $\mathrm{~A} 2$ & $\mathrm{Bu} 7$ \\
\hline Frequency $\left(\mathrm{cm}^{-1}\right)$ & 1047 & 1113 & 1305 & 1318 & 2184 \\
\hline$\phi_{1}$ (high value) & .07 & .02 & .13 & .21 & 1 \\
\hline$\phi_{2}$ (low value) & .02 & .02 & .03 & .05 & .16 \\
\hline
\end{tabular}

These different efficiencies in the isomerization process are probably due to the existence of two trapping sites for the molecule. Different constraints are exerted in each site thus leading to different couplings with the external modes (hindered translation or rotation) of the molecule. These low frequency modes are probably strongly involved in the relaxation process by helping the matching of the energy for a quasi-resonant intramolecular V-V transfer. The high $\phi$ site probably corresponds to the weaker coupling with the localized modes. The deactivation of the excited mode is indeed slower and this promotes the transfer to the specific excited state allowing the isomerization.

In the case of the Au8 excitation, the very weak reaction rate can be explained by the existence of the A8 band of the gauche form high-frequency shifted by $1.5 \mathrm{~cm}^{-1}$ but with an absorption cross-section about ten times lower. The spectral width of the CLIO laser does not allow to separate the two excitations and the global absence of isomerization effect proves that the gauche $->$ trans process is much more efficient than the trans $\rightarrow$ gauche process for the same excitation energy.

Nevertheless, except for the $v_{12}$ and the $v_{8}$ excitations and within the experimental uncertainties, $\phi$ shows a monotonous increase with the energy given to the system. This is consistent with a statistical distribution of this energy within all the vibrational modes (RRKM behavior [11]). This increase is observed for the two sites : the main mechanism leading to the isomerization is actually due to the 
intramolecular structure while its velocity depends on intermolecular interactions. Calculations are currently on progress to fit these data with the prediction of RRKM theory.

\section{CONCLUSION}

These first results on the selective infrared excitation of different modes of the $\mathrm{CH}_{2} \mathrm{D}-\mathrm{CH}_{2} \mathrm{D}$ molecule isolated in a xenon matrix show that most of the vibrational modes are efficient to induce the isomerization. The analysis of the reaction velocities shows the importance of the environment of the molecule and confirms the participation of the localized modes in the vibrational relaxation processes. Although the reaction rate generally grows with energy, a non RRKM behavior is observed in the case of the $v_{12}$ and the $v_{8}$ excitations. Further developments are underway to confirm our interpretation. In particular, the calculation of the localized modes and of the anharmonic terms of the molecular potential will give quantitative information about the intra- and inter- molecular couplings.

Aknowledgments : We gratefully aknowledge Mr. E. Chipon and the UNICAM company for a technical support.

\section{REFERENCES}

[1] Legay F., Chemical and Biochemical Applications of Lasers (ed. C. Bradley Moore, Academic Press, 1977) pp. 43-86.

[2] Baldeschwieler J. D. and Pimentel G. C., J. Chem. Phys. 33 (1960) 1008-1015.

[3] McDonald P. A. and Shirk J. S., J. Chem. Phys. 77 (1982) 2355-2364.

[4] Poliakoff M. and Turner J. J., Chemical and Biochemical Applications of Lasers (ed. C. Bradley Moore, Academic Press, 1980) pp. 175-216.

[5] Frei H. and Pimentel G. C., Ann. Rev. Phys. Chem. 36 (1985) $491-524$.

[6] Räsänen M., Kunttu H. and Murto J., Laser.Chem. 9 (1988) 123-146 (and references therein).

[7] Prazères R., Berset J. M., Glotin F., Jaroszinski D. and Ortéga J. M. Nucl. Instr. and Meth. A331 (1993) 15-19.

[8] Moazzen-Ahmadi N., Gush H. P., Halpern M., Jagannath H., Leung A. and Ozier I., J. Chem. Phys. 88 (1988) 563-577.

[9] Fernández-Sánchez J. M., Gómez P. C. and Montero S., J. Chem. Phys. 99 (1993) 1575-1590.

[10] Roubin P., Kakou R., Verlaque P., Monnier M., Pourcin J. and Bodot H., Chem. Phys. Lett. 160 (1989) 345-349.

[11] Gilbert R.G. and Smith S.C., Theory of Unimolecular and Recombination Reactions (Blackwell Scientific Publications, 1990). 